\title{
Development of Rapid SPECT Acquisition Protocol for Myocardial Perfusion Imaging
}

\author{
A. Hans Vija, Member, IEEE, Johannes Zeintl, Student Member IEEE, James T. Chapman, Eric G. Hawman, J. \\ Hornegger, Members, IEEE
}

\begin{abstract}
Advances in reconstruction techniques and instrumentation improve image quality. Improved image quality could either improve confidence and diagnostic ability, or increase throughput, and lower injected dose. Current imaging guidelines give insuffient consideration to advances in iterative reconstruction methods that include collimator modeling, attenuation, and scatter correction, and best matched acquisitions. Thus advanced reconstruction is often sub-optimally used in a clinical setting. In this work, we first characterize the effects of various acquisition and reconstruction protocols with the explicit aim to reduce scan time without adverse affects as compared to FBP driven protocols. For this we characterize image features, such as nonuniformity and wall thickness of the cardiac insert inside the large anthropomorphic torso phantom (Data Spectrum) and correlate them with human observer ROC results. When reconstructing the data with OSEM with 3D collimator and detector response compensation ("Flash3D") we found that the detection ability is not impacted when using $6^{\circ}$ angular steps, and thus reducing the acquisition time by $50 \%$, as compared to the current method. A further reduction can be achieved if the rest study is scanned in the continuous instead of the step-and-shoot mode (10\%). Dwell time can also be reduced slightly; however the myocardial count density should not be below at least $1 \mathrm{cnt} / \mathrm{mm}^{2}$ for rest and summed stress. Clinical trials need to confirm the findings.
\end{abstract}

\section{INTRODUCTION}

A DVANCES in reconstruction techniques and instrumentation improve image quality. Improved image quality could either improve confidence and diagnostic ability, or increase efficiency. Current imaging guidelines [1,2] are well adjusted for FBP reconstruction but give insufficient consideration to advances in iterative reconstruction methods that include collimator modeling, attenuation, and scatter correction. Literature provides some advise on how to set the various acquisition and reconstruction parameters for some applications [3-10]. However, in clinical practice subtle differences from these studies exist, which are introduced by the specifics of the entire image formation chain including the patient and the tracer uptake.

Manuscript received November 17, 2006. Asterisk indicates corresponding author.

*A. Hans Vija (e-mail: hans.vija@siemens.com), James T. Chapman, Eric G. Hawman are with Siemens Medical Solution USA, Inc., Molecular Imaging, Hoffman Estates, IL 60192, USA.

Johannes Zeintl is a student with the University of Erlangen-Nürnberg, Institute of Pattern Recognition, Erlangen, Germany. He is currently with Siemens Molecular Imaging.

Joachim Hornegger is with University of Erlangen-Nürnberg, Institute of Pattern Recognition, Erlangen, Germany.
An iterative reconstruction method, such as Ordered Subset Expectation Maximization (OSEM) with 3D Collimator and Detector Response Correction (CDRC), and optional attenuation and scatter compensation ("Flash3D") requires some additional considerations prior to an acquisition to obtain best reconstruction results. The term "best" should be understood in the context of a specific imaging task of a specific clinical application and clinical practicality. The images are eventually read by a physician, drawing medical information from them, so we want to employ human observer study methods. We use a Receiver-Operating-Characteristics (ROC) study to assess the performance and estimate the Area Under the Curve (AUC) [11]. However unlike in most studies, e.g. $[12,13]$ we use phantom data measured with clinical system.

The aim of this work is to develop an acquisition protocol for SPECT cardiac imaging for reduced scan time without adversely affecting the clinical read. The best suited protocol is then tested in clinical trials.

\section{MEthOD}

\section{A. Overview}

We propose to work with measured data acquired with a clinical system instead of Monte Carlo simulation, which can only approximate a real clinical system. From this measured data ("reference"), we create "what-if" datasets. For this we developed a DICOM compatible tool which extracts angular views from a projection data set, rebins the matrix and pixel size and reduces counts in a pixel to some fraction $\mathrm{f}$ using binomial subsampling, and maintaining the Poisson character of the data [15]. On purpose we don't allow for projection view interpolation as such data would not correspond to data acquired with our systems.

This method can be applied to both phantom and patient data. For instance, this allows the clinician to test non-standard protocols on retrospective data prior to applying such a modified protocol prospectively to patients. There are of course limitations to this approach, which will be addressing later.

We obtained 12 standard clinical same-day protocol data to assess the image quality from "what-if" acquisitions by visual inspection from an e.cam (6) and a c.cam (6) with 64-by matrix, $6.6 \mathrm{~mm}$ (e.cam), $6.4 \mathrm{~mm}$ (c.cam), 90 detector 
configuration, $180^{\circ}$ arc, 64 projections $\left(2.8^{\circ}\right) \mathrm{NCO}, 140 \mathrm{keV}$ centered, 20\%. Rest: 25s/view; Stress: 20s/view.

We quantitatively characterize image features of the cardiac inserts inside the large anthropomorphic phantoms made by Data Spectrum, Hillsborough, NC using an in-house tool [14]. This tool also allows both the researcher and the practitioner in the clinic to perform advanced application driven quality control to assess the entire image formation chain of a system or protocol.

In this work we show ROC results using data from the large anthropomorphic static male phantom with one ("abnormal") or no lesion ("normal") in the cardiac insert. We acquired a "reference phantom database" on the Siemens Symbia ${ }^{\circledR}$ T6. The acquisition is performed with all our available low energy parallel-hole collimators with $15 \%$ peak window, centered at $140 \mathrm{keV}$, and $15 \%$ lower scatter window abutting the peak window. However, here we only present results from the LEHR collimators.

\section{B. Phantom preparation and acquisition}

The details of the phantom loading and acquisitions are shown in TABLE I and TABLE II. The phantom was acquired from $45^{\circ}$ left posterior oblique to $45^{\circ}$ right anterior oblique with $90^{\circ}$ head configuration.

TABLE I: LOADING PARAMETERS FOR LARGE ANTHROPMORPHIC TORSO BY DATA SPECTRUM

\begin{tabular}{cccc}
\hline \hline & $\begin{array}{c}\text { Injected } \\
\text { Activity }(\mathrm{mCi})\end{array}$ & $\begin{array}{c}\text { Concentration } \\
(\mu \mathrm{Ci} / \mathrm{ml})\end{array}$ & $\begin{array}{c}\text { Concentration } \\
\text { Ratio }\end{array}$ \\
\hline Myocardium & 2.0 & 18.2 & 14.0 \\
Defect & 0.05 & 15.9 & 12.3 \\
Liver & 12.0 & 10.4 & 8.0 \\
Background & 12.0 & 1.3 & 1.0 \\
\hline \hline
\end{tabular}

TABLE II: ACQUISITION PARAMETERS

\begin{tabular}{lc}
\hline \hline Head Separation Angle & $90^{\circ}$ \\
Scan Range & $180^{\circ}$ \\
Angular Step & $1^{\circ}$ \\
Orbit & non-circular \\
Pixel Size $(\mathrm{mm})$ & 1.65 \\
Matrix Size & $256 \times 256$ \\
Total Counts $(\mathrm{kc})$ & 90000 \\
Count Density in myocardium $\left(\mathrm{cts} / \mathrm{mm}^{2}\right)$ & $44-52$ \\
\hline \hline
\end{tabular}

For the abnormal scan we introduce the smallest available $45^{\circ}$ extent defect $(4.7 \mathrm{ml})$ in the inferior wall at the mid-position at a concentration of about $87.5 \%$ of the myocardium. The defect center is at 6 o'clock, close to the position of the well-known 5 o'clock artifact usually induced by the lack of attenuation correction. The extent in the reconstructed volume of both the lesion and the 5 o'clock artifact is about equal.
From these "reference high count" data sets we derive a number of other projection datasets by sub-sampling counts (binomial), angular projections (extraction), matrix (pixel) size (interpolation), and orbit range (extraction).

\section{Reconstruction}

Subsequently data is reconstructed with Flash-3D, our OSEM with 3D CDRC, and also with FBP for reference (TABLE III). Our analysis package is then used to quantitatively characterize image features, such as non-uniformity, wall thickness or infero-lateral artifact of the myocardial VolumeOf-Interest [14].

TABLE III: RECONSTRUCTION PARAMETERS USING FLASH3D

\begin{tabular}{cccccccc}
\hline \hline Total \\
Counts & $\begin{array}{c}\text { Counts } / \mathrm{v} \\
\text { iew }\end{array}$ & $\begin{array}{c}\text { Count density } \\
\text { in } \\
\text { myocardium } \\
\text { (cts/mm2) }\end{array}$ & $\begin{array}{c}\text { Angular } \\
\text { step }\left(^{\circ}\right)\end{array}$ & Iterations & Subsets & $\begin{array}{c}\text { \# OSEM } \\
\text { Updates }\end{array}$ & $\begin{array}{c}\text { Post- } \\
\text { smoothing } \\
\text { (FWHM in } \\
\text { pixel) }\end{array}$ \\
\hline \multirow{4}{*}{$900 \mathrm{k}$} & $15 \mathrm{k}$ & $0.45-0.70$ & 3 & 5 & 12 & 60 & 2 \\
& $30 \mathrm{k}$ & $0.90-1.40$ & 6 & 10 & 6 & 60 & 2 \\
& $45 \mathrm{k}$ & $1.50-1.80$ & 9 & 15 & 4 & 60 & 2 \\
\hline \multirow{4}{*}{$450 \mathrm{k}$} & $7.5 \mathrm{k}$ & $0.23-0.35$ & 3 & 6 & 6 & 36 & 2 \\
& $15 \mathrm{k}$ & $0.45-0.70$ & 6 & 12 & 3 & 36 & 2 \\
& $22.5 \mathrm{k}$ & $0.75-0.90$ & 9 & 18 & 2 & 36 & 2 \\
\hline \multirow{4}{*}{$450 \mathrm{k}$} & $7.5 \mathrm{k}$ & $0.23-0.35$ & 3 & 6 & 12 & 72 & 2 \\
& $15 \mathrm{k}$ & $0.45-0.70$ & 6 & 12 & 6 & 72 & 2 \\
& $22.5 \mathrm{k}$ & $0.75-0.90$ & 9 & 18 & 4 & 72 & 2 \\
\hline & $7.5 \mathrm{k}$ & $0.23-0.35$ & 3 & 12 & 12 & 144 & 2 \\
$450 \mathrm{k}$ & $15 \mathrm{k}$ & $0.45-0.70$ & 6 & 24 & 6 & 144 & 2 \\
& $22.5 \mathrm{k}$ & $0.75-0.90$ & 9 & 36 & 4 & 144 & 2 \\
\hline \hline
\end{tabular}

Note: A post-smooth with a 3D Gaussian with a FWHM of 2 pixels is applied in all cases.

\section{Observer study and analysis}

For the ROC study multiple realizations are generated from the low-noise, but not noiseless reference data. The generation of $\mathrm{M}$ realizations all with $\mathrm{k}$ counts from data with $\mathrm{K}$ counts can be regarded as essentially uncorrelated if binomial subsampling is used and $\mathrm{k} / \mathrm{K}<<1$, the error of the variance is then proportional to $\mathrm{k} / \mathrm{K}$. We generate $\mathrm{N} / 2$ normal and $\mathrm{N} / 2$ abnormal sets $(\mathrm{N}=100)$ for the ROC study.

The data was presented to 6 readers. We used nonparametric estimation of the AUC and standard error [11] and then bootstrap (100 repeats/reader) to determine unbiased estimates and standard errors.

\section{RESULTS}

The results of the ROC study are summarized in TABLE IV , and Fig. 1 depicts the ROC curves for $3^{\circ}$ and $6^{\circ}$ angular sampling at a total count of $450 \mathrm{kc}$ and 900kc. TABLE V shows the numerical characterization of the nonuniformity (nu) and its standard error (nu_se) in \% as well as the FWHM of the wall thickness (wt) in $\mathrm{mm}$ and its standard error (wt_se). 
Fig.2 is a complex graph attempting to combine the information in TABLE IV and TABLE $\mathbf{V}$ and thus correlating detectability from an observer study as measured by AUC with a numerical characterization of an image feature, such as the myocardial nonuniformity for the acquisition protocols using $3^{\circ}, 6^{\circ}$, and $9^{\circ}$ angular sampling at total counts of $450 \mathrm{kc}$ and $900 \mathrm{kc}$ at increasing iteration updates $(36,72$, and 144$)$ using Flash3D. The 2D error bars correspond to standard errors of the AUC and nonuniformity respectively. In Fig.3 we plot the standard error of the AUC for all readers using bootstrap on the ordinate against the nonuniformity showing a minimum of the standard error around 11\%. In Fig.4 we show a graph which not only relates AUC and nonuniformity but also recovered resolution by measuring the FWHM of the wall thickness at $450 \mathrm{kc}$. The left ordinate shows nonuniformity and AUC on the same scale, the right ordinate the FWHM of the reconstructed wall thickness $(\mathrm{mm})$ and the abscissa shows the number of OSEM updates. The more updates the better the resolution recovery, yet nonuniformity worsens. However the AUC remains stable and worsens once nonuniformity worsens above some threshold which in this case is around 72 updates, which as Fig. 3 shows is at about $11 \%$.

Fig.5 depicts the standard error as it trends with the decrease in infero-lateral intensity for $3^{\circ}, 6^{\circ}$, and $9^{\circ}$ angular sampling at total counts of $450 \mathrm{kc}$ and $900 \mathrm{kc}$. The intensity decreases from $-17 \%$ to $-20 \%$ with decreased total counts, while the standard error increases from $2.5 \%$ to $3.5 \%$. The slope is essentially the same for $3^{\circ}$ and $9^{\circ}$ angular sampling, but for $6^{\circ}$ the range of intensity change is compressed and thus the slope is steeper.

Fig.6 depicts in a waterfall graph the potential acquisition time savings. If the standard protocol with $3^{\circ}$ angular steps in stepand-shoot represents $100 \%$, then the acquisition time could be reduced to $36 \%$ if instead a protocol with $6^{\circ}$ angular step size in continuous mode with an $80 \%$ dwell time reduction is used.

Fig.7 and Fig.8 show example sagittal images of the cardiac insert inside the phantom and of a heart from clinical data processed in such a way as to demonstrate resulting image quality of "what-if" protocols. Fig.7 shows an example images comparing a standard protocol and a protocol using Flash3D in $36 \%$ of the acquisition time. The infero-lateral artifact due to lack of attenuation correction is well visible in both images. Fig. 8 shows in image (A) and (C) the original projection data, but reconstructed with FBP and Flash3D. The projection data in (B) is extracted from the original and represents a "what-if" protocol data set at twice the angular step size and $80 \%$ dwell time reduction, reconstructed with Flash3D.
TABLE IV: ROC AUC (USING BOOTSTRAP)

\begin{tabular}{ccccc}
\hline \hline $\begin{array}{c}\text { Total Counts } \\
(\mathrm{Kc})\end{array}$ & OSEM updates & $\begin{array}{c}\text { Angular step } \\
\left({ }^{\circ}\right)\end{array}$ & AUC & SE \\
\hline \multirow{3}{*}{450} & & 3 & 0.88 & 0.04 \\
& \multirow{2}{*}{36} & 6 & 0.89 & 0.03 \\
\cline { 3 - 5 } 900 & & 9 & 0.90 & 0.03 \\
\hline \multirow{3}{*}{450} & & 6 & 0.93 & 0.03 \\
& & 9 & 0.95 & 0.02 \\
& & 3 & 0.93 & 0.03 \\
\hline \multirow{2}{*}{450} & 6 & 0.91 & 0.03 \\
& & 9 & 0.91 & 0.03 \\
& 144 & 3 & 0.91 & 0.03 \\
\hline \hline
\end{tabular}

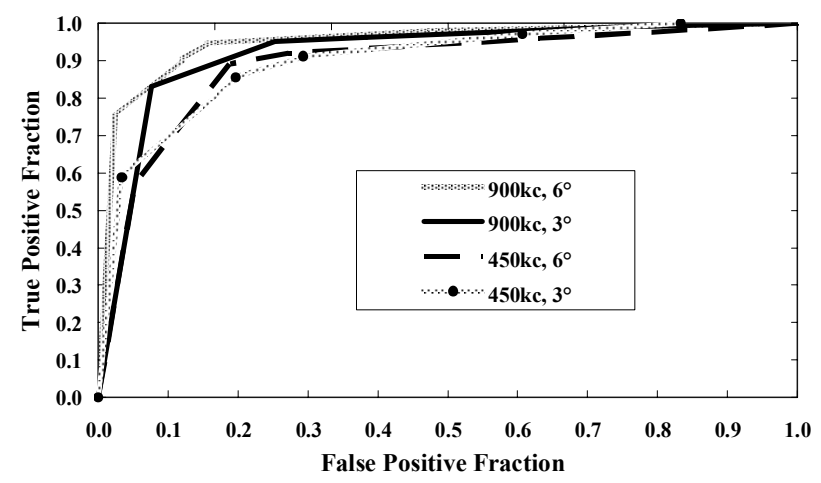

Fig.1. ROC curves for $3^{\circ}$ and $6^{\circ}$ angular steps at $450 \mathrm{kc}$ and $900 \mathrm{kc} .3^{\circ}$ and $900 \mathrm{kc}$ represents standard acquisition and $6^{\circ}$ and $450 \mathrm{kc}$ corresponds to a scan in $50 \%$ of the acquisition time, but same dwell time as the standard scan.

TABLE V: NumERICAL CHARACTERIZATION OF IMAGE FEATURES

\begin{tabular}{|c|c|c|c|c|c|c|}
\hline $\begin{array}{c}\text { Total counts } \\
(\mathrm{Kc})\end{array}$ & OSEM updates & $\begin{array}{c}\text { Angular step } \\
\left({ }^{\circ}\right)\end{array}$ & $\mathrm{NU}(\%)$ & SE_NU (\%) & WT (mm) & $\begin{array}{c}\text { SE_WT } \\
(\mathrm{mm})\end{array}$ \\
\hline \multirow{3}{*}{450} & \multirow{6}{*}{36} & 3 & $9.7 \%$ & $0.1 \%$ & 23.9 & 0.05 \\
\hline & & 6 & $10.3 \%$ & $0.1 \%$ & 23.9 & 0.07 \\
\hline & & 9 & $10.0 \%$ & $0.1 \%$ & 23.9 & 0.06 \\
\hline \multirow{3}{*}{900} & & 3 & $9.4 \%$ & $0.1 \%$ & 21.7 & 0.04 \\
\hline & & 6 & $9.5 \%$ & $0.1 \%$ & 21.7 & 0.04 \\
\hline & & 9 & $9.2 \%$ & $0.1 \%$ & 21.8 & 0.04 \\
\hline \multirow{3}{*}{450} & & 3 & $10.7 \%$ & $0.2 \%$ & 21.1 & 0.05 \\
\hline & 72 & 6 & $11.0 \%$ & $0.1 \%$ & 21.3 & 0.05 \\
\hline & & 9 & $10.7 \%$ & $0.2 \%$ & 21.2 & 0.05 \\
\hline \multirow{3}{*}{450} & & 3 & $12.3 \%$ & $0.2 \%$ & 19.9 & 0.06 \\
\hline & 144 & 6 & $12.2 \%$ & $0.1 \%$ & 19.8 & 0.04 \\
\hline & & 9 & $12.3 \%$ & $0.2 \%$ & 19.8 & 0.05 \\
\hline
\end{tabular}




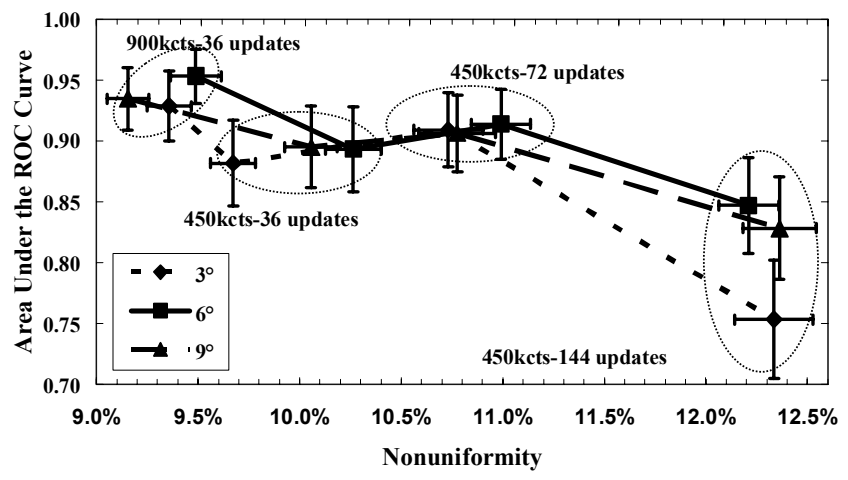

Fig.2. Correlating detectability with a measure for myocardial non-uniformity for $3^{\circ}, 6^{\circ}, 9^{\circ}$ angular sampling, 36, 72 and 144 OSEM updates and 2 total count levels at $450 \mathrm{kc}$, and $900 \mathrm{kc}$. The 2D error bars show standard error of AUC and nonuniformity respectively.

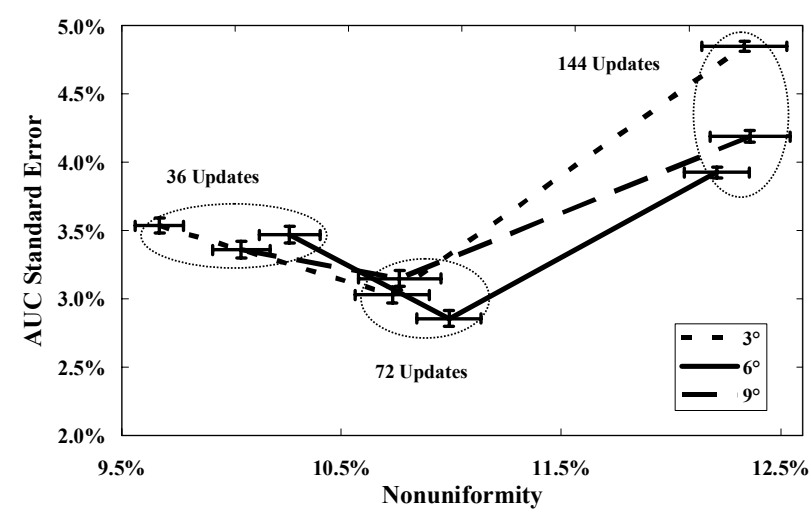

Fig.3. The standard error of the AUC using bootstrap is correlated with nonuniformity.

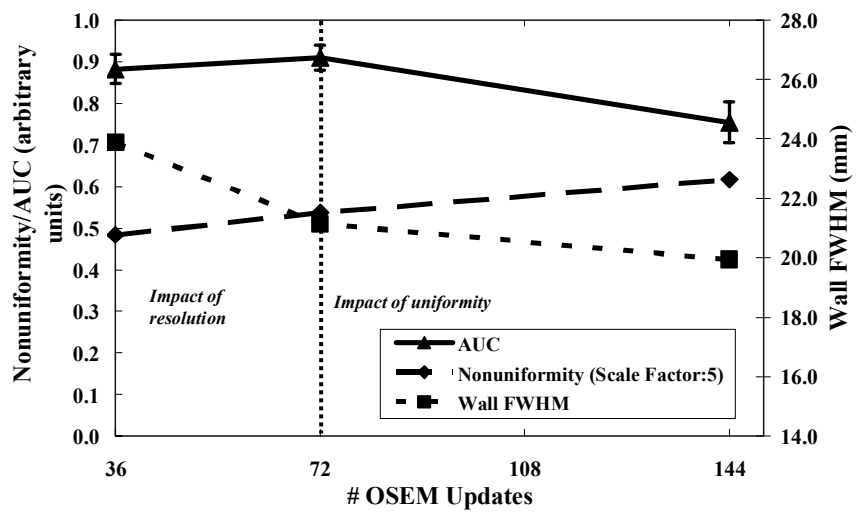

Fig.4. The left ordinate shows nonuniformity and AUC on the same scale and the right ordinate the FWHM of the reconstructed wall thickness $(\mathrm{mm})$ and the abscissa shows the number of OSEM updates. The more updates the better the resolution recovery, yet nonuniformity worsens. However the AUC remains stable and worsens once nonuniformity worsens above some threshold which in this case is around 72 updates.

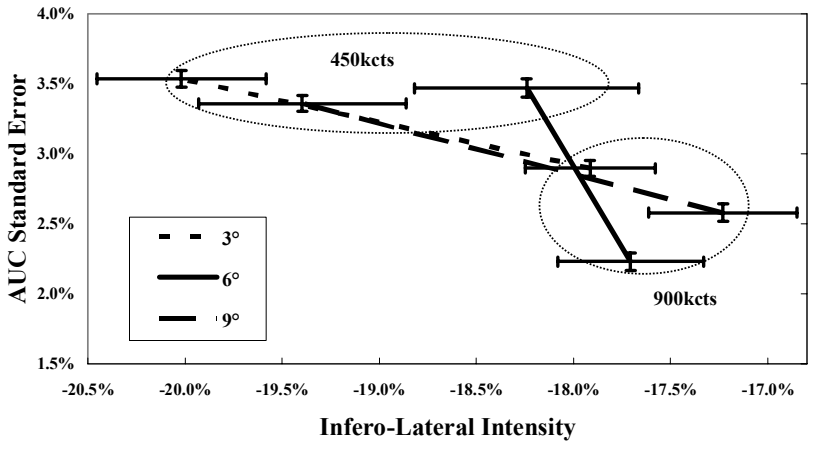

Fig.5. The standard error of the AUC using bootstrap is correlated with a numerical characterization of the infero-lateral intensity decreases for $3^{\circ}, 6^{\circ}$, $9^{\circ}$ angular sampling and the 2 total count levels of $450 \mathrm{kc}$, and $900 \mathrm{kc}$.

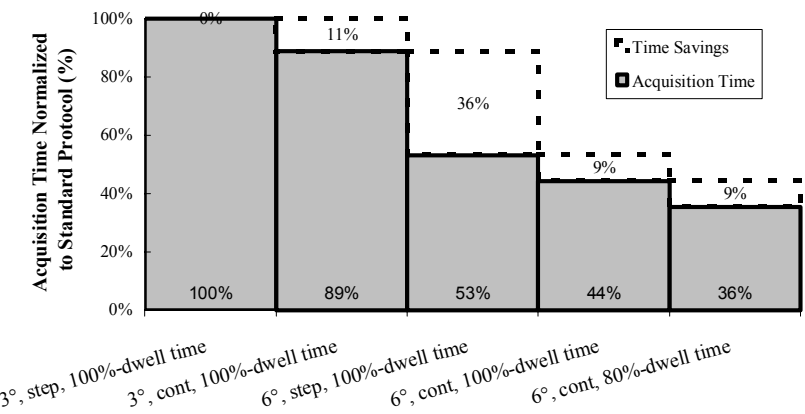

Fig.6. Breakdown of the acquisition time and potential savings depending on the acquisition protocol using LEHR collimators. Acquiring in continuous instead of step-and-shoot mode saves about $10 \%$.

Torso Phantom, normal (33kcts/view; 64², $4.8 \mathrm{~mm}$ )

(A) FBP $\left(3^{\circ}\right.$, step, $>1.1 / \mathrm{mm}^{2}$ )

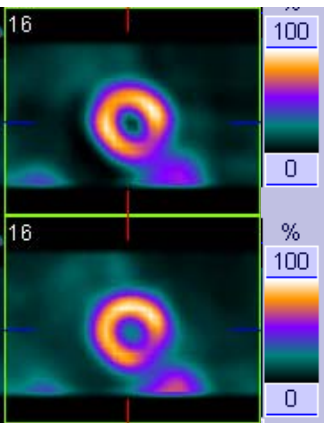

Fig.7. Example images comparing a standard protocol and a protocol using Flash $3 \mathrm{D}$ in $36 \%$ of the acquisition time. The infero-lateral artifact due to lack of attenuation correction is well visible. 


\section{Example Patient data (rest, $64^{2}, 6.6 \mathrm{~mm}$ )}

\author{
(A) FBP $\left(2.8^{\circ}\right.$, step, 25 \\ sec/view) \\ $20 \mathrm{sec} / \mathrm{view})$
}

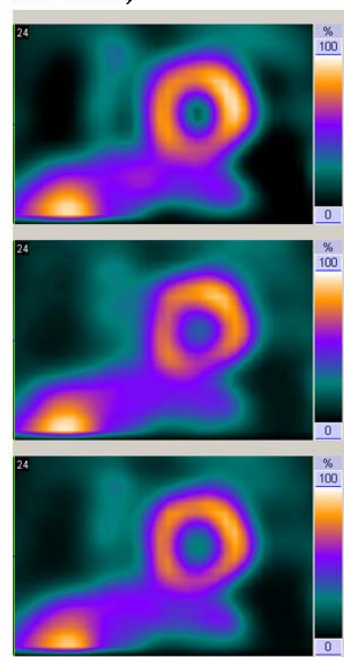

Fig.8. Example patient data. For images (A) and (C) the original projection data is used, but reconstructed with FBP and Flash3D. The projection data in (B) is extracted from the original data and represents a "what-if" protocol data set with twice the angular step size and $80 \%$ dwell time reduction, and reconstructed with Flash3D.

\section{DISCUSSION}

We acquired cardiac phantoms with various parallel-hole collimators and modified the data sets corresponding to various protocols and reconstructed them with Flash3D. Features of the phantom images were numerical characterized and related to the AUC of ROC studies. For instance, in Fig.2 - Fig.5 we show how the AUC and its standard error trends with nonuniformity, wall thickness and the intensity of the infero-lateral region ("5 o'clock" artifact).

The increased reader variability as measured by the standard error of the AUC may have its roots in a decrease of confidence to make a detection decision. However, this statement is at this point very speculative and primarily based on the individual experiences of the observers. We have placed the lesion close to the 5 o'clock artifact on purpose to a) make the task more challenging, but b) to also compare with attenuation correction in a future study. Resolution recovery improves with increasing number of updates but nonuniformity increases as well. Both effects decrease the infero-lateral intensity and thus increase the contrast of the 5 o'clock artifact with respect to the myocardial wall. The reader variability shows a minimum at about $11 \%$ of nonuniformity with a resolution recovery of FWHM $=20 \mathrm{~mm}$ at 72 updates which is twice the actual wall thickness of 10 mm (Fig.3, Fig.4).

It seems plausible that the interplay between resolution and nonuniformity are the key drivers for this particular detection task in this application. The results indicate that nonuniformity impacts detection performance more significantly that resolution.
These findings also correspond to feedback from physicians who usually like to see a smooth myocardium yet with the best possible resolution. Our phantom studies indicate that a threshold for nonuniformity exists, however we don't know how the non-uniformity and the wall thickness measures will translate to clinical patient data.

Creating data from "what-if" scenarios and subjecting the images to human observers and numerical analysis proved to be an efficient method. It correlates the detection performance with image feature characterization. At this point we don't believe that simple phantom-based measures such as the nonuniformity which relies on knowing the shape of the myocardium can predict detection performance. However, it can be used for ranking and to exclude protocols which for instance would show a higher nonuniformity value than the some threshold (here 11\%) and thus obviating the need to assess the performance of that protocol with an expensive observer study. The count level is not clinical and the AUC is generally too high as compared to clinical data, but at this point we are interested in comparing and ranking system performance.

This method is also suited for existing clinical data and the use in a clinical setting. Even retrospective objective image analysis studies could be performed if there is follow-up information on such patient data.

However, there a several caveats: for instance, we traded-off the full flexibility of a simulation for working with measured data, and thus were constrained by the experimental set-up.

We could not test a population of lesions of various extents. Even the smallest lesion available to us at the low contrast of $88 \%$ and total counts of about $8 \%-10 \%$ of actual clinical counts was rather easily detected (AUC $>0.85$ ). It has been shown that a population of objects with unknown lesion position results in AUC closer to clinical reality $(\approx 0.75-0.85)$ [16]. However we believe that despite the non-clinical count level and lack of object variability the approach reveals comparative information between various protocols.

The multiple realizations of binomially sub-sampled data needed for the ROC study is derived from the low-noise (but not noiseless) measured reference phantom dataset. This introduced an error as the realizations are not statistically independent. The error is however small if the sub-sampling fraction $\mathrm{f} \ll 1$.

If ROI count density is below a certain threshold nonuniformity becomes too high $(>11 \%)$ even at low number of updates, limiting resolution recovery, and decreasing overall detection performance. The range of the threshold seems to be between $0.3-0.8 \mathrm{cntc} / \mathrm{mm}^{2}$, but this needs further investigation. In practice, $1 \mathrm{cnt} / \mathrm{mm}^{2}$ as the lower threshold 
may be a good number to keep in mind. FBP generally performed worse than OSEM-3D, but more analysis is needed.

\section{CONCLUSION}

Current recommendation for SPECT cardiac imaging of spatial and angular sampling, and count density may well be matched to FBP, but not to OSEM-3D.

When imaging the cardiac phantom with a $45^{\circ}$ extent lesion of about $5 \mathrm{ml}$ in the cardiac insert we found that $3^{\circ}$ sampling offers only little more resolution recovery as for instance $6^{\circ}$ steps do and only little more advantage in the detection ability when the data is reconstructed with OSEM-3D. Our isotropic resolution recovery resolves the target structures well even up to about $9^{\circ}$ angular steps.

However one has to keep in mind that minimizing nonuniformity was the driving image quality feature, as physicians usually express discontent with a "blobby" myocardium, even if the myocardial walls are better resolved. The ROC results also indicate that the detection ability in the phantom myocardium is mostly driven by the nonuniformity and only second by resolution.

In rest studies, the continuous as compared to step-and-shoot mode could further reduce acquisition time by $\sim 10 \%$. Acquisition time per view (dwell time) can be reduced as long as the count density is not below at least $1 \mathrm{cnt} / \mathrm{mm}^{2}$ in the rest or summed stress projection of the myocardial ROI. Typically one can expect a reduction of dwell time to $\sim 80 \%$ as compared to the standard protocol. In a gated study the count density in the myocardium ROI in one gate could fall below 1 $\mathrm{cnt} / \mathrm{mm}^{2}$, in which case the reconstruction parameter for OSEM-3D should be adjusted by lowering the number of updates, and by even reverting to ML-EM. In general resolution recovery can only occur if enough counts are available to justify it. At very low counts and count density one cannot expect good resolution recovery.

We developed SPECT cardiac acquisition protocols, using our OSEM-3D ("Flash3D") where the acquisition time could be reduced to between $1 / 3$ and $1 / 2$ of currently suggested FBPdriven acquisition protocols. Prospective clinical studies are being conducted to assess the performance of a protocol using $6^{\circ}$ angular sampling, and thus reducing acquisition time by $50 \%$.

\section{ACKNOWLEDGMENT}

The authors thank the participants in the observer study as well as Günter Hahn for valuable discussion and the Systems Test team at Hoffman Estates for their help during the acquisition of the data.

\section{REFERENCES}

[1] B. Hesse et al, "EANM/ESC procedural guidelines for myocardial perfusion imaging in nuclear cardiology", EJNM, 32(7), 2005, p.855
[2] FJ Kocke et al.," ACC/AHA/ASNC Guidelines for the Clinical Use of Cardiac Radionuclide Imaging", ACC/AHA/ASNC Practice Guidelines, 2003

[3] RE Moorin, "Optimization of technetium-99m Sestamibi single-photon emission tomography to define multidrug resistance with confidence", NMC 25(10), 2004,p. 1039

[4] MK O'Connor, CB Hruska, "Effect of tomographic orbit and type of rotation on apparent myocardial activity.",NMC 26(1), 2005, p.25

[5] J Chen, et al.,"Optimized acquisition of processing protocols for I-123 cardiac SPECT imaging",13(2),JNC, 2006, p. 1

[6] C Kamphuis, FJ Beekman, MA Viergever, "Evaluation of OS-EM vs. ML-EM for 1D, 2D, and fully 3D SPECT re-construction", IEEE TNS, 44(3), 1996, p.2018

[7] Y Takahasji, et al.,"Evaluation of the number of SPECT projections in the ordered subsets-expectations maximization image reconstruction, Ann. Nuc. Med. 17(7), 2003, p.525"

[8] JA Bieszk, EG Hawman, "Evaluation of SPECT Angular Sampling Effects: Continuous Versus Step-and-Shoot Acquisition”, JNM 28, 1987, p.1308

[9] MW Groch, WD Erwin, "SPECT in the Year 2000: Basic Principles." Journal of Nuclear Medicine Technology Volume 28(1), 2000, P233244

[10] YH Lau, BF Hutton, FJ Beekman, "Choice of collimator for cardiac SPET when resolution compensation is included in iterative reconstruction,", European Journal of Nuclear Medicine and Molecular Imaging, 28(1), 2001, p.39

[11] J.A. Hanley, B.J. McNeil, "The Meaning and Use of the Area under a Receiver Operating Characteristic (ROC) Curve", Radiology 143: 29-36, April 1982

[12] S. Sankaran, E.C. Frey, et al, "Optimum Compensation Method and Filter Cutoff Frequency in Myocardial SPECT: A Human Observer Study", JNM 43, 2002, p.432

[13] M.V. Narayanan, M.A. King, et al, "Human Observer ReceiverOperating-Characteristic Evaluation of Attenuation, Scatter, and Resolution Compensation Strategies for $99 \mathrm{mTc}$ Myocardial Perfusion Imaging", JNM 44, 2003, p.1725

[14] J.T. Chapman, J. Zeintl, A.H. Vija, et al. "A Quantitative Method for Assessing Performance of Cardiac Imaging Systems", M06-284, MIC2006

[15] J. Zeintl, A.H. Vija, J.T. Chapman, et al. "Quantifying the Effects of Acquisition Parameters in Cardiac SPECT Imaging and Comparison with Visual Observers", M14-303, MIC 2006

[16] B.M.W. Tsui, et al. "Effect of Object Variability in Observer Performance Studies for Image Quality Assessment”, M03-1, MIC2006 